Esta revista forma parte del acervo de la Biblioteca Jurídica Virtual del Instituto de Investigaciones Jurídicas de la UNAM

\title{
LESLIE GREEN*
}

\section{1. ¿Por qué se interesó inicialmente en la filosofía del derecho?}

Llegué a la filosofía del derecho cuando descubrí que los problemas de la filosofía política que yo más quería explorar requerían de una mejor comprensión de la naturaleza del derecho.

Mis intereses filosóficos se desarrollaron gradualmente. El tema no era enseñado en la escuela secundaria, aunque yo tenía un dedicado profesor de historia que me puso varios libros en mi camino, entre ellos Lenguaje, verdad y lógica de Ayer. Fue el primer libro de filosofía que leí. Aunque había partes que me parecían oscuras y poco atractivas, admiraba su crudeza y su brío. Probablemente, él me inoculó en contra de las tonterías que un novato eventualmente encontrará en los estantes de "filosofía" de una librería o de una biblioteca pública. Como estudiante de pregrado, estudié política, así como filosofía, pero eran temas paralelos, porque mi trabajo en política en aquella época era fundamentalmente institucional y empírico. Mi primer ensayo como estudiante de filosofía política fue sobre la pregunta de si Rousseau era un totalitario o era liberal. No puedo recordar lo que argumenté —espero que haya tenido el buen sentido de resistir las alternativas-. Un poco después, leí En defensa del anarquismo de Robert Paul Wolff, que tuvo un efecto muy inquietante: sus conclusiones me parecieron tanto irresistibles como inaceptables, sus argumentos me preocuparon por mucho tiempo.

Mi interés cada vez más se fue dirigiendo a esos temas, y, cuando me gradué, solicité el ingreso a instituciones de posgrado donde pudiera dedicarme más a ellos, y también a escuelas de derecho, en caso de que necesitara un trabajo. Cuando me ofrecieron una beca para ir a Oxford, la decisión fue fácil.

Dejé Canadá por Inglaterra antes del primer referéndum sobre la independencia de Quebec y del periodo de intenso debate sobre la reforma constitucional que le siguió. Incluso desde la distancia era imposible no verse atrapado por todo esto, lo que incluía las preguntas de si Canadá debía tener una carta de derechos atrincherada

* Oxford University, leslie.green@law.ox.ac.uk. Publicado originalmente en inglés en Morton, E. Nielsen J. (ed.), Legal Philosophy Five Questions (Automatic Press, 2007). 
Esta revista forma parte del acervo de la Biblioteca Jurídica Virtual del Instituto de Investigaciones Jurídicas de la UNAM

\section{LESLIE GREEN}

y, si era así, cuáles derechos deberían estar en ella (al final, Canadá adoptó una solución que concordaba con mi propia ambivalencia: una carta de derechos atrincherada supervisada por las cortes, pero sujeta a derogación temporal por el Poder Legislativo). Estos asuntos estaban mucho en mi mente, aunque todavía no escribía sobre ellos. En esta etapa, mi formación intelectual estaba más influida por los filósofos a mi alrededor que por mi reacción a los eventos políticos. Hart, Honoré, Raz, Dworkin y Finnis estaban activos en la filosofía del derecho, al igual que Taylor, Sen, Parfit, Mackie y MacDowell en la filosofía moral y política. Era una comunidad extraordinaria de académicos.

Fui muy afortunado con mis primeros profesores, especialmente con Jonathan Glove, Joseph Raz y Charles Taylor, quienes no habrían podido ser mejores, o menos parecidos filosóficamente. Estudié filosofía del derecho, en primer lugar, con Raz en 1980. Recuerdo muchas de esas primeras tutorías. Una fue acerca de la individuación de las normas"; la pregunta, como Bentham la formuló, de qué constituye una norma completa. Ésta era una cuestión en la que Raz había puesto un esfuerzo prodigioso; sin embargo, por mis lecturas, sabía que otros, incluyendo Dworkin, se burlaban de toda la idea de la individuación de las normas - ¿qué estábamos tratando de hacer, contarlas? - A algunos, la idea les parecía una ociosa pieza de metafísica (es interesante, por tanto, leer en La justicia con toga que Dworkin ahora ha logrado individuar incluso conceptos de derecho y ha contado cuatro de ellos). Me esforcé por entender el significado del problema y decir algo útil al respecto. Lo único que se me ocurrió fue una desatinada analogía con la idea de Chomsky de una estructura profunda en el lenguaje, exhibiendo así mi ignorancia en dos temas. Raz demolió mi analogía - tranquila, pero rotundamenteen pocos minutos. Mis largas noches de trabajo habían producido algo más que un metafórico replanteamiento del problema. Fue una experiencia educativa y una lección de humildad.

Por fuera de las tutorías, me lancé a leer de cerca la Teoría general del derecho y el Estado y la Teoría pura del derecho de Kelsen, La moralidad del derecho de Fuller y, por supuesto, El concepto de derecho de Hart. Otros libros que ahora están en el canon, por entonces todavía estaban frescos, y, como lo hacen los estudiantes de pos- 
Esta revista forma parte del acervo de la Biblioteca Jurídica Virtual del Instituto de Investigaciones Jurídicas de la UNAM

LESLIE GREEN

grado, nosotros también inspeccionábamos los nuevos trabajos de Dworkin, Raz y Finnis, esperando encontrar nuevas ideas y errores. En los seminarios, Raz estaba ya empezando a presentar las principales ideas de La moral de la libertad, y Dworkin estaba hablando sobre el material que se convertiría en El imperio de la justicia. Podíamos ver que grandes cosas estaban pasando, era excitante estar envuelto incluso como espectadores. También aprendí mucho de otros estudiantes. Jeremy Waldron y yo trabajamos la Filosofía del derecho de Hegel en nuestros desayunos, Denise Réaume me forzó a tomar en serio la teoría feminista, la cual luego influenció mi trabajo sobre género y sexualidad, Anthony Teasdale me insistió en no perder de vista las realidades empíricas e institucionales de la política. Como teníamos completa libertad de asistir a las clases, además de mi trabajo para los cursos, asistí a varías clases en filosofía general y derecho sustantivo, las cuales ahora tengo que enseñar yo mismo.

Luego de sobrevivir los extenuantes exámenes, decidí empezar mi doctorado. Taylor me dirigió durante las etapas iniciales de mi trabajo acerca de las perspectivas comunitarias de la autoridad; sin embargo, mi interés y aproximación se distanció de la suya, y cuando Taylor estaba a punto de renunciar a la cátedra Chichele, pregunté a Raz si desearía tomarme como un estudiante investigador. Afortunadamente, él había perdonado (o más probablemente olvidado) mi ensayo sobre la individuación de las normas y aceptó convertirse en mi supervisor. Para mí, en todo caso, esos fueron años extremadamente placenteros. Gracias a los buenos resultados de mis exámenes y a varios capítulos de mi disertación, conseguí un trabajo en el Lincoln College, en Oxford, donde descubrí qué tanto me gustaba enseñar, y donde empecé a desear que hubiera una posibilidad de ganarme la vida con esta actividad. Aunque mi periodo in statu pupillari ${ }^{1}$ había llegado a su final, mi educación continuó cuando me uní a los grupos de discusión que se reunían en las aulas de Tony Honoré, en All Souls. Mi primera ponencia criticó una de las doctrinas centrales de Finnis. La evidente perplejidad de Finnis contribuyó poco a mi propia confianza, pero para agravar mis ansiedades, Hart

1 La expresión in statu pupillari es usada para referirse a los estudiantes de posgrado en los primeros años, antes de obtener un título de maestría (N. del T.). 
Esta revista forma parte del acervo de la Biblioteca Jurídica Virtual del Instituto de Investigaciones Jurídicas de la UNAM

\section{LESLIE GREEN}

se quedó dormido a los tres minutos de iniciada mi intervención, aunque se despertó al final con una inquisitiva pregunta a la que añadió, al día siguiente, una carta, que vigorosamente continuaba con el tema. No podía entender por qué Hart estaba tan preocupado sobre mis objeciones a Finnis. Todavía no había visto los esbozos de lo que sería el post scriptum a El concepto de derecho, donde, en un esfuerzo por evadir las contundentes críticas de Dworkin a su teoría de las obligaciones, Hart dio un giro convencionalista, que era vulnerable a las mismas objeciones que ahora yo formulaba en contra de Finnis. En algún momento de aquella época me di cuenta de que me estaba estableciendo en la carrera de filósofo del derecho.

2. ¿Por cuál de sus contribuciones a la filosofía jurídica hasta ahora le gustaría más ser recordado, y por qué?

Cualquier filósofo de mediana edad o más joven que sea moderadamente optimista tiene el derecho de esperar a que su mejor trabajo esté por delante. Dicho eso, sin embargo, tengo dudas sobre el deseo de ser recordado por el trabajo propio, o por alguna cuestión en absoluto. Marco Aurelio ha dicho con razón que toda la fama termina en el olvido. Comparto, además, el punto de vista de Séneca de que la vida es suficientemente larga, siempre que mantengamos la calma y no perdamos nuestro tiempo. Aún más, el deseo de ser recordado puede convertirse en un vicio si fomenta una disposición de escribir para ser recordado. Así que lucho para mantener la fe en la antigua tesis de que los filósofos tienen que estar sólo orientados a lo que es verdadero e iluminante y no a ser inolvidables o, incluso, originales. No es necesario decir que es un estándar que difícilmente puede inspirar una satisfacción duradera con los escritos propios o con los de muchos otros. Con esto, no quiero decir que no tengo un orgullo especial por algunos de mis escritos, pero eso tiene que ver menos con alguna supuesta recordación que con los retos intelectuales (y en ocasiones personales) que significó escribirlos. De cualquier modo, si quisiera ser recordado, no estoy seguro si sabría cómo empezar la campaña. Algunos temas que fueron terriblemente difíciles de hacer bien atraen poca atención, otros fueron escritos con facilidad, y aún así terminaron reeditados, en antologías, tradu- 
Esta revista forma parte del acervo de la Biblioteca Jurídica Virtual del Instituto de Investigaciones Jurídicas de la UNAM

cidos, y así sucesivamente. Imagino que los postulados llamativos y tontos tienden a ser recordados por unos pocos años, pero este no es el tipo de filosofía que ninguno de nosotros debería aspirar a escribir.

Deberíamos responder esta pregunta más ampliamente, para incluir también las propias contribuciones a la formación de otros filósofos del derecho. Siempre he tenido una especial admiración por aquellos profesores que, tanto por misión o irresistible ejemplo, moldearon las futuras generaciones de pensadores en sus campos, aquellos que les hicieron ver por qué un tema importa y por qué vale la pena dedicarse a esos temas. Recuerdo con nostalgia aquellos que lo hicieron por mí, y espero que algunos de mis propios estudiantes puedan llegar a sentirse de la misma manera. De esta forma, supongo, me alejo de mis ancestros estoicos.

3. ¿Cuáles son los temas más importantes de la filosofía del derecho, y por qué son temas distintivos de ésta y no de cualquier otra disciplina?

La noción de "importancia" es dependiente del interés, y la relación "más importante que" no está conectada al dominio de los temas relevantes, de modo que no hay temas "más importantes" en la filosofía del derecho. En lo que respecta a su especificidad, muchas disciplinas, incluyendo varias ramas de la filosofía, tienen una custodia conjunta sobre los principales temas de la disciplina.

Un tema es importante cuando da lugar a algunas perplejidades serias, lo que nos deja perplejos depende tanto de cosas externas a la filosofía como de cosas internas a ella. En los años sesenta, la independencia de las antiguas colonias británicas intensificó muchas perplejidades acerca de la continuidad del derecho a través del tiempo. Hoy, es difícil hacer que los estudiantes vean por qué estos temas son (o fueron) muy importantes. En los setenta y ochenta, hubo un interés enorme por la justicia, los derechos y la igualdad, que se fue perdiendo a medida que el compromiso por la justicia social en los Estados capitalistas ricos se fue desvaneciendo. Ahora estamos enmedio de un periodo de intenso interés sobre el estado de derecho, el constitucionalismo y la globalización. Estas reflexio- 
Esta revista forma parte del acervo de la Biblioteca Jurídica Virtual del Instituto de Investigaciones Jurídicas de la UNAM

nes también resultarán ser productivas, y luego, muy probablemente, también pasarán. Tales cambios en la atención filosófica son benignos en la mayoría de los casos, y, de cualquier forma, son inevitables. Ellos también, sin embargo, nos vuelven cautelosos en declarar que algo es "el más importante" tema en la filosofía del derecho.

Dicho esto, considero que el intento de ofrecer una explicación sobre la naturaleza del derecho (y problemas afines) reviste cierta importancia. Esto revela mi posición acerca de la forma en la que otros problemas en la filosofía del derecho, incluyendo problemas normativos, necesitan ser abordados. Piense en la antigua cuestión de si existe una obligación general de obedecer al derecho ¿el hecho de que algo sea requerido por el derecho añade alguna consideración moral adicional a favor del acatamiento, una que fortalezca o reemplace nuestras razones ordinarias que dependen del contenido de la norma y que sujete a todos los sujetos jurídicos en todas las ocasiones en las cuales la obediencia es requerida?

Así las cosas, probablemente notará que mostrar que existe tal obligación es una batalla muy difícil (¿en todas las ocasiones?, ¿aplicadas a toda la gente?). Así que ¿por qué no simplemente disminuimos las expectativas y decimos que hay una obligación de obedecer al derecho siempre que haya una razón moral para obedecer, digamos, en siete ocasiones de diez, o una que aplique a la persona promedio? ¿Por qué insistir en la universalidad? Porque eso es lo que el derecho nos pide. Él pretende determinar el rango de nuestra obligación de obedecer, él fija la línea entre el incumplimiento lícito y la desobediencia civil. Ahora bien, ésa es una tesis de la teoría general del derecho. Si tiene sentido pensar que el derecho nos hace ese tipo de demandas y qué contenido damos a sus demandas, son temas sobre la naturaleza del derecho. Cuestiones similares surgen con respecto a otros importantes problemas, incluyendo la naturaleza del razonamiento jurídico y el valor del estado de derecho.

También son importantes las ramas de la jurisprudencia especial - los intentos por encontrar explicaciones filosóficas satisfactorias de los fundamentos de la responsabilidad civil, de los contratos, del derecho penal, los derechos humanos, etcétera-. Algunos problemas en el área, tales como la causación, la responsabilidad y el consentimiento atraviesan muchas de estas ramas; otros están limita- 
Esta revista forma parte del acervo de la Biblioteca Jurídica Virtual del Instituto de Investigaciones Jurídicas de la UNAM

dos a un campo doctrinal particular. Aquí, el trabajo del filósofo del derecho necesita cooperar de forma más estrecha con el del abogado académico, porque es una vergüenza para la teoría de la responsabilidad civil, por ejemplo, que lo que declare ser la verdadera esencia de la responsabilidad civil sea algo que de hecho es marginal a la ley realmente efectiva, o que algo que la teoría sobre los contratos declare como un caso limítrofe sea considerado por las cortes como un contrato perfectamente normal.

Aparte de la propia temática, no estoy seguro de qué tanto estos temas son particulares sólo de la filosofía del derecho, si son temas sobre los cuales otras ramas de la filosofía o de los estudios sociales no puedan arrojar una luz importante. Mi propia posición es que la filosofía del derecho es una rama de la filosofía política en sus aspectos normativos e institucionales, y que hay sólo límites aproximados entre la filosofía política y los otros aspectos de la filosofía práctica. A diferencia de Kelsen, por tanto, espero que una correcta filosofía del derecho sea muy "impura". No obstante, aun cuando tiene sentido pensar en algunos de estos temas como propiedad privada de la filosofía del derecho, todavía necesitamos entender la conexión entre ellos y otros temas: como insistía Hart, una larga parte de la filosofía del derecho consiste en explicar las relaciones entre el derecho y otras formas de orden social, y entre el derecho y la moral. Nadie tiene el monopolio sobre estos temas.

4. ¿Cuál es la relación entre la filosofía del derecho y la práctica jurídica?, ¿los filósofos del derecho deberían preocuparse más acerca del efecto de sus enseñanzas en la práctica jurídica?

Estoy tentado a contestar "nada" o "en absoluto", y éstas casi que son las respuestas correctas. Obviamente, depende de lo que uno entienda por "práctica jurídica". Si esto significa simplemente aquellas prácticas que son jurídicas, entonces la relación es que la filosofía del derecho ofrece una explicación filosófica de ellas, en la jurisprudencia especial y general. No obstante, la mayor parte de las personas que hacen esta pregunta están en realidad interesadas en un tema bastante diferente: ¿cuál es la relación entre la filosofía del derecho y la práctica del derecho, i. e. el ejercicio del derecho? 
Esta revista forma parte del acervo de la Biblioteca Jurídica Virtual del Instituto de Investigaciones Jurídicas de la UNAM

\section{LESLIE GREEN}

Aquí la dificultad está en que ejercer el derecho incluye un variado y complejo conjunto de prácticas, de las cuales no todas tienen mucho que ver con el derecho o no son objetos fértiles para la investigación filosófica. Ellas incluyen no sólo la litigación y la adjudicación, sino también (y más a menudo) entrevistar, aconsejar, negociar, regatear, llevar la contabilidad, etcétera (incluso, he oído de una facultad de derecho que contrata a personas para que enseñen a sus estudiantes cómo vestirse para el éxito. Asumo que vestirse es también parte de la práctica jurídica). Cada abogado practicante sabe que el éxito en su profesión tiene tanto que ver con la destreza en estas cosas como con todo lo que podríamos considerar perspicacia jurídica. Cada profesor de derecho conoce estudiantes débiles que se convirtieron en brillantes y acaudalados abogados. Esto sugiere que Aristóteles tenía razón al mirar la investigación y la abogacía como dos empresas radicalmente diferentes, lo cual podría explicar el porqué abordó los principales problemas de la filosofía del derecho en la Ética y la política, en tanto que confinó el ejercicio del derecho a la Retórica. Ese me parece un enfoque adecuado. Lo que el abogado quiere, sobre todo, es ganar una disputa, y, por lo tanto, necesita estar exquisitamente atento a las predisposiciones de su audiencia actual; el filósofo lo que quiere, sobre todo, es tener un argumento, y desea sólo persuadir a los razonables.

Aquellos que moldean su filosofía del derecho esperando que sea útil a los abogados litigantes o a otros, por tanto, necesitan adaptarse a las características parroquiales del sistema jurídico en cuestión. Si eres del tipo de filósofo que aspira a una larga e influyente audiencia, estarás tentado a adaptarla a las predisposiciones de las cortes de países grandes e influyentes. No veo que se saque mayor provecho de esto. En ocasiones, puede llevar a una deshonestidad intelectual; siempre conduce a inexactitudes.

Déjenme dar un ejemplo. Los estadounidenses del siglo XXI tienen que vivir bajo una Constitución de los siglos XVII y XIX, en un país con débiles partidos políticos y una cultura influenciada por formas de cristianismo decadentes que están prácticamente extinguidas en las democracias maduras. Ésas no son circunstancias favorables para los grupos minoritarios, y especialmente no lo son para las lesbianas y los homosexuales. Sus cortes federales son, por tanto, 
Esta revista forma parte del acervo de la Biblioteca Jurídica Virtual del Instituto de Investigaciones Jurídicas de la UNAM

muy tolerantes con la discriminación por la orientación sexual, aunque ellas pretenden ver con malos ojos la discriminación por razón del género. Por ello, los abogados liberales estadounidenses algunas veces alegan que la discriminación a la orientación sexual sólo es discriminación por razón del género. Es posible imaginar cómo la historia continúa: el género juega un rol en definir la orientación sexual, porque un homosexual está atraído a una persona de su mismo género, y la orientación sexual se entrecruza con la discriminación de género en la medida en que ella dañe a la gente que no se ajusta a las normas aceptadas (incluyendo normas de conducta sexual) para la gente de ese género. A menos que haya un cambio radical en la cultura de Estados Unidos, un argumento en este sentido es probablemente la mejor opción en una corte estadounidense. No obstante, sería muy extraño ${ }^{2}$ ofrecer ésta como una explicación de la naturaleza de la discriminación por la orientación sexual. Las prohibiciones a los matrimonios y a las adopciones por parte de parejas del mismo género no benefician a un género sobre el otro; ellas no restringen las opciones de la gente en relación con su género, y mientras la señal de la discriminación por el género es a la mujer, la señal de la discriminación por la orientación sexual es, en cambio, a la "transformación". Centrarse en el hecho de que aquellos que discriminan por este motivo emplean inevitablemente una clasificación con base en el género - como si la antidiscriminación fuera sólo un tabú acerca de ciertas clasificaciones- es pasar por alto enteramente lo que está mal con la discriminación. Aún más, es parroquial. Si lleváramos tal caso a una corte canadiense o sudafricana, nunca ofreceríamos algo tan rebuscado como la teoría género-reduccionista, que puede ser la última y mejor esperanza del abogado estadounidense, pero las Constituciones de estos países en realidad prohíben la discriminación por motivos de orientación sexual. Por supuesto, esto muestra que la Constitución estadounidense necesita ser reformada, pero, como mis amigos estadounidenses me recuerdan constantemente, esta idea y 3.50 dólares pueden conseguirte un

2 Green utiliza la expresión queer, que significa en inglés tanto homosexual como extraño (N. del T.). 
Esta revista forma parte del acervo de la Biblioteca Jurídica Virtual del Instituto de Investigaciones Jurídicas de la UNAM

\section{LESLIE GREEN}

chai-soy-latte, si tienes tanta suerte como para vivir en algún lugar de Manhattan, Austin o Berkeley.

La cuestión es esta. Los abogados tienen pocas opciones distintas a trabajar con o alrededor de lo que las fuentes les dejan y lo que la política del día a día les permite - pero ¿por qué debería un filósofo elegir trabajar con tal arnés?-. No puedo dejar de sentir que el deseo de hacer esto revela el error de no asumir una importante verdad sobre la naturaleza del derecho. La fuerza de los argumentos jurídicos siempre depende de las fuentes, la fuerza de los argumentos filosóficos nunca depende de ellas. Las cortes pueden reírse de usted y usted puede estar precisamente en lo correcto. Las cortes pueden adularle y usted puede estar completamente equivocado (y puede, como Kelsen, llegar a arrepentirse del día en que sea citado).

Por estas razones, no creo que deberíamos gastar tiempo preocupándonos por el efecto que nuestras investigaciones puedan tener en la práctica jurídica. Aún más: los abogados no necesitan más ayuda de los filósofos del derecho que la que los científicos prácticos necesitan de los filósofos de la ciencia, o los artistas necesitan de los filósofos de la estética. Cuando las cortes citan a los filósofos del derecho es usualmente por razones decorativas y no por razones funcionales - todo lo que los más extremos realistas del derecho dijeron acerca de los argumentos de autoridad usados por las cortes es absolutamente cierto acerca de sus vergonzosas injerencias en la filosofía del derecho-. Desde luego, la filosofía del derecho contribuye en formas indirectas a nuestra cultura jurídica y política, pero es sólo un vector de influencia, y su efecto neto es pequeño y difícil de predecir.

5. ¿A qué problema, tema o rama de la filosofía del derecho le gustaría que se prestara más atención en el futuro?

En primer lugar, necesitamos mucho más trabajo sobre los proble-mas familiares, los cuales están siendo constantemente reformulados en respuesta a los desarrollos de la filosofía general y a los cambios en nuestra cultura intelectual y política. Aquí hay dos ejemplos. Independientemente de lo que el derecho sea, incluye reglas jurídicas. En cada sistema jurídico hay algunas reglas jurídicas 
Esta revista forma parte del acervo de la Biblioteca Jurídica Virtual del Instituto de Investigaciones Jurídicas de la UNAM

LESLIE GREEN

que no fueron creadas por actos cuyo objetivo era hacer una regla, así que no llegaremos muy lejos sin una teoría de las reglas consuetudina-rias. Ahora bien, piense en todas las diferentes formas en que hemos tratado de explicarlas: la teoría práctica de las reglas, explicaciones interpretativistas, de la teoría de juegos, del razonamiento práctico. Ciertamente, hemos hecho grandes avances en este tema, al mostrar que algunas teorías no son ni siquiera candidatas plausibles, pero al mismo tiempo continuamos regresando al problema cada vez que se presentan nuevas herramientas filosóficas o cuando se imponen nuevas perspectivas. A causa de lo primordial que las reglas son para muchas áreas de la filosofía y la teoría social, podemos estar confiados en que éste será un problema recurrente.

Otro ejemplo: por un largo periodo de tiempo se ha creído que el realismo jurídico ha estado tan muerto como cualquier teoría pueda estarlo. Al igual que las teorías imperativas del derecho, hacía parte de la prehistoria de nuestra disciplina. Es verdad que una forma ingenua del realismo siguió siendo popular en algunas facultades de derecho y en algunos departamentos de ciencia política, pero no porque hubiera alguna convincente respuesta a las objeciones de Kelsen o Hart. Sobrevivió por la ignorancia y por una pérdida de interés en los asuntos que lo llevan a uno a leer sus libros. No obstante, cuando el naturalismo filosófico llegó a la filosofía del derecho a través del trabajo de Brian Leiter, el realismo tuvo una segunda oportunidad como una protociencia de la decisión judicial - una primitiva, pero, para algunos, correcta en su dirección metodológica y con más probabilidades de producir un conocimiento confiable sobre la naturaleza del derecho que cualquier tipo de análisis conceptual o interpretativo-. Ahora bien, este debate está lejos de ser superado; de hecho, apenas está comenzando. Lo que quiero decir es que ahora necesitamos reformular y reestructurar nuestro entendimiento de las fortalezas y debilidades del realismo jurídico. Un debate que parecía resuelto, superado, agotado, ahora está reemergiendo con nuevos términos. Lo mismo sucede en otras áreas de la filosofía del derecho. El cambio casi siempre profundiza nuestra comprensión de los temas, y cuando tenemos suerte, nos acerca a la verdad sobre ellos. 
Esta revista forma parte del acervo de la Biblioteca Jurídica Virtual del Instituto de Investigaciones Jurídicas de la UNAM

\section{LESLIE GREEN}

Así que el primer paso es llevar a los viejos problemas cualesquiera que sean los nuevos recursos y las nuevas perspectivas que parezcan plausibles. No obstante, también hay problemas a los cuales no les hemos prestado la atención debida. El primero es sustantivo. Quisiera que los filósofos del derecho, y no sólo los historiadores y sociólogos, prestaran mucha más atención al papel del poder en el derecho (y por 'poder' aquí quiero decir poder social, no poderes normativos, tales como el poder de legislar o contratar -ya prestamos suficiente atención a éstos-). Este problema aparece de muchas formas. Primero, un sistema jurídico no existe a menos que sea ampliamente eficaz, lo cual significa que pueda realmente influenciar la conducta y a través de ella los intereses de la gente. Segundo, el poder social es relevante para la atribución de la responsabilidad jurídica. Tercero, los sistemas jurídicos dependen de instituciones centralizadas que dan a algunas personas poderes sobre otras, como Hart célebremente señaló, al separar la existencia de las normas jurídicas de una amplia aceptación social de las normas sociales.

Creo que hay dos razones por las que la filosofía del derecho contemporánea rehúye estos temas. La primera es el éxito rotundo de Hart y Raz en atacar las teorías que fundamentan la normativa del derecho en la coerción. Es como si habiendo refutado la tesis de que cada norma o cada sistema jurídico necesariamente despliegan un poder coercitivo los filósofos del derecho decidieran que no hay nada más que merezca la pena decirse sobre el poder en el derecho: derecho no es poder, y ése es el final de la historia. Soy consciente, por supuesto, de que los académicos inspirados por Foucault dicen que están interesados en el poder. No obstante, no espero mucho de este enfoque, al menos en su presente forma. Cuando aborda al derecho, despliega una posición terriblemente anticuada sobre lo que es la teoría jurídica, repitiendo viejos argumentos en contra de las teorías de la soberanía y las teorías imperativas que Hart hizo ya hace una generación. Además, la concepción foucaultiana de poder es muy amplia, contando prácticamente cada causación como una forma de poder. Esto alienta pensamientos sorprendentes, pero vacíos, de que el poder está en todas partes, que el discurso está "censurado" por las reglas de la gramática, y así sucesivamente. Si eso fuera todo lo que el poder representara, podríamos olvidarnos de él 
Esta revista forma parte del acervo de la Biblioteca Jurídica Virtual del Instituto de Investigaciones Jurídicas de la UNAM

\section{LESLIE GREEN}

con seguridad. Sin embargo, ésa es una explicación defectiva, queda por hacer un buen trabajo para reemplazarla. Los filósofos políticos han estado pensando profundamente sobre este tema por un largo periodo; es hora de que los filósofos del derecho se unan en este debate.

Una segunda razón de nuestro olvido del poder es la reticencia de los filósofos del derecho a participar como debieran en las preguntas empíricas e institucionales. Aquí vemos el legado de cierto estilo de filosofía. G. A. Cohen relata una historia sobre sus días en Oxford durante los "sesenta", cuando era obligatorio atacar un argumento poco claro con la pregunta de si se proponía una tesis conceptual o (simplemente) empírica. A la agotadora objeción "Pero eso es sólo una cuestión empírica", Cohen recuerda a uno de sus colegas implorando "Podemos tener posiciones empíricas algunas veces?"3 Yo tengo simpatía por esta súplica, porque creo que la filosofía del derecho seria depende de afirmaciones empíricas sobre las instituciones políticas y la sicología humana, aunque a un nivel muy gene-ral. Aun Hart ocasionalmente sucumbió al ethos antiempírico; alguna vez declaró que el hecho de que el homo sapiens necesitara comida para sobrevivir era "meramente contingente". En sus más cuidadosos estados de ánimo, sin embargo, vio que también necesitamos ocuparnos de las verdades que no son ni conceptualmente necesarias ni meramente contingentes, sino que son, podríamos decir, no accidentes. Es decir, dada la naturaleza de la gente y la naturaleza del derecho, algunas cosas son esperadas en el normal curso de los eventos, a no ser que algo inusual intervenga. Así que mientras no es una verdad necesaria que los sistemas jurídicos deberían incorporar concepciones comunes de responsabilidad, no es un accidente que así lo hagan. Tampoco es un accidente que el derecho sea una forma jerárquica de organización social que, además de ser un sistema normativo, es un sistema de poder social. ¿Deberíamos excluir estos temas del campo de la teoría jurídica, porque ellos no son conceptualmente necesarios? Hart no lo habría hecho. Él estaba interesado en las verdades necesarias acerca del derecho, pero su idea de

3 Cohen, G. A., If you're an Egalitarian. How Come you're so Rich?, Cambridge, Harvard University Press, 2000, p. 185. 
Esta revista forma parte del acervo de la Biblioteca Jurídica Virtual del Instituto de Investigaciones Jurídicas de la UNAM

lo que ellas comprendían era muy católica; incluía no sólo las necesidades conceptuales, sino también las naturales e, incluso, las que podríamos llamar necesidades culturalmente dependientes. Podemos, obviamente, repetir el mantra vacío de que las verdades necesarias deben ser verdades para todos los sistemas jurídicos posibles. De cierta forma, esto es correcto. El problema está en que es difícil hacerse una idea de todos los sistemas jurídicos posibles, a menos que eso signifique todos los sistemas jurídicos imaginables, lo cual deja a la filosofía del derecho presa de los poderes variables de imaginación de la gente (algunos dirán que ellos no pueden imaginarse un sistema jurídico sin sanción — para mí, eso es un juego de niños comparado con tratar de imaginar un sistema jurídico en el cual todas las condiciones de verdad de las proposiciones jurídicas sean también condiciones de justificación de la coerción jurídica-. Más sobre este tema luego). Tengo la sensación de que deberíamos prestar más atención a las necesidades "blandas" del derecho, a lo que he venido llamando como verdades "no-accidentales". Creo que entre ellas encontraremos verdades conceptuales y empíricas muy importantes acerca del poder que podrían ayudar a traernos de regreso a una conversación provechosa con el resto de la teoría social y política.

El segundo tema es metodológico, o quizá submetodológico: ¿cuáles son los criterios de idoneidad de una teoría del derecho? Necesita ser inteligible y coherente, pero hay una diferencia entre una teoría del derecho que es meramente consistente y otra que es correcta. Uno puede consistentemente representar un sistema jurídico como si fuera un sistema de incentivos (conozco algunos filósofos del derecho que niegan esta tesis - les deseo suerte probando su posición-). Tenga en cuenta que no digo que un sistema jurídico sea un sistema de incentivos, o que puede ser así representado sin alguna pérdida. Sin embargo, uno puede representar consistentemente los sistemas jurídicos de formas muy diversas: como un conjunto de órdenes emitidas por un soberano unitario, como un conjunto de órdenes a los jueces para que apliquen sanciones cuando la gente se porta mal, como ordenanzas de la razón para el bien común, y así sucesivamente. Ninguna de ellas da cuenta de todos los aspectos; cada teoría jurídica aprieta y recorta de alguna forma. Como Robert Nozick 
Esta revista forma parte del acervo de la Biblioteca Jurídica Virtual del Instituto de Investigaciones Jurídicas de la UNAM

LESLIE GREEN

inolvidablemente dijo, la explicación filosófica es como presionar y lanzar objetos aleatorios en un perímetro fijo de forma específica, algunas veces cortando las esquinas y aun abandonando objetos a tiempo para una fotografía rápida del arreglo ordenado — sujeto a retoques posteriores-.

Algunas tesis sobre el derecho sucumben ante la semántica: el derecho no es un organismo o un elemento. Otras son epistémicamente irresponsables: no podemos más concebir teorías que requie-ren referencias a espíritus o entidades no existentes, o que se basen en métodos que requerirían su existencia. Y, sin duda, queremos evitar teorías patentemente tontas, tales como aquellas que nos comprometen con la posición para la cual el derecho es indiferente entre que sus sujetos se abstengan de asesinar, o asesinen, pero sufran la pena. No obstante, entre las teorías libres de estos crasos errores todavía nos confrontamos con elecciones que pueden ser justificadas sólo a la luz de los criterios de idoneidad de una teoría jurídica, y aquí no hemos hecho mucho todavía.

Kelsen pensó que una teoría del derecho tenía que ser pura, independiente de cualquier tesis acerca de la moral, la sociología o las ciencias empíricas. Hart dijo que había que capturar con fidelidad "el punto de vista interno", y así entender las funciones del derecho y de las reglas desde la perspectiva de los que de hecho las usan. Finnis dijo que se debería mostrar cómo el derecho es obligatorio prima facie; Dworkin, cómo todas las proposiciones verdaderas del derecho funcionan al mismo tiempo como justificaciones de la coerción oficial. Raz dijo que la filosofía del derecho debía consistir sólo de proposiciones acerca del derecho que sean necesariamente verdaderas, explicativas y que se ajusten a la tesis de las fuentes. Estos criterios son constantemente invocados, pero insuficientemente defendidos. Para tomar sólo un ejemplo, el argumento de Dworkin de la no neutralidad de la filosofía del derecho depende de su opinión, según la cual el argumento jurídico tiene que mostrar cómo lo que acepta como derecho ofrece una razón moral prima facie para la coerción, y que ninguna razón de este tipo sería válida a menos que esté permitida por estándares establecidos de antemano (bajo una muy especial interpretación de "establecidos" y una especial interpretación de "de antemano"). No obstante, muchas personas recha- 
Esta revista forma parte del acervo de la Biblioteca Jurídica Virtual del Instituto de Investigaciones Jurídicas de la UNAM

\section{LESLIE GREEN}

zan esa condición de idoneidad. No todos piensan que la gente tiene derecho a ganar un caso jurídico sólo si tienen un derecho establecido de antemano, muchos creen que lo que hace verdadera a una proposición sobre la ley no necesariamente se refiere a la pregunta de si alguien tiene un derecho a coaccionar a la gente a some-terse a esa ley, aun menos si tienen un deber de obedecer. Este no es el lugar para explorar estos temas, sino solamente para decir que hemos pasado la etapa de la filosofía del derecho en la que cualquie-ra de nosotros podía dar esas tesis por descontadas o, mucho menos, como si existiera una meseta de acuerdos. Nuestro periodo de inocencia metodológica ha pasado; ahora necesitamos defensas explícitas de estas posiciones, $y$, preferiblemente, una defensa que no haga una explicación de la naturaleza del derecho completamente ajena a la explicación de la naturaleza de otras instituciones sociales importantes tales como los partidos políticos, las cárceles o las familias. En cualquier caso, éstos son algunos de los temas que creo será fructífero explorar más adelante. 